\title{
An Introduction to Semigroup Theory
}

J. M. Howie

$$
\text { 1976, } x+284 \text { pp., } £ 9.80 / \$ 24.75 \quad 0.12 .356950 .8
$$

Any short book on semigroup theory would inevitably be shallow were its scope not limited to certain parts of the theory. This volume concentrates primarily on regular semigroups, for which a well developed and fairly cohesive theory exists. In particular, the longest chapter is devoted to inverse semigroups, which have been-and continue to be-perhaps the most rewarding area of study.

\section{Matroid Theory \\ D. J. A. Welsh \\ 1976, $x i i+428$ pp., $£ 15.00 / \$ 38.00 \quad 0.12 .744050 . X$}

This book attempts to show the unifying and central role which matroids have played in combinatorial theory over the past decade. The first half is an introduction to matroid theory in which most theorems are proved or an exact reference is given. The second places the reader at the frontier of the of the subject-at this level, finding it impossible to prove every result, the author has treated in some detail the more important topics. The work contains many exercises and unsolved problems and there is a comprehensive bibliography.

\section{Subharmonic Functions Volume 1 W. K. Hayman, FRS}

$1976, x v i i i+284$ pp., $£ 11.60 / \$ 25.50 \quad 0.12 .334801 .3$

The study of subharmonic functions as a generalistion of logs of regular functions started as a systematic discipline about thirty years ago, with the work of Ahlfors, Heins and Kjellberg. The present volume, the first since then to develop this viewpoint systematically, steers an intermediate course between books on various aspects of abstract potential theory and books on function theory using some potential theoretic techniques. The author covers the classical theory of subharmonic functions as well as some applications: to ensure that it is as self-contained as possible he has included a comprehensive preface to the book as a whole, and shorter introductions to each chapter.

\section{Academic Press}

\section{London New York San Francisco}

A Subsidiary of Harcourt Brace Jovanovich, Publishers

24-28 Oval Road, London NW1, England

111 Fifth Avenue, New York, NY 10003, USA

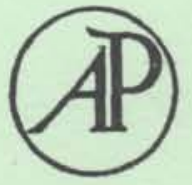

